www. revistadyo.com

\title{
Impacto de la Perspectiva de Género en la Resiliencia de la Cadena de Suministro
}

\author{
Llanos Cuenca, Ana Esteso, Elena Navarro \\ Recibido: 12 de Octubre de 2018 / Aceptado: 21 de Noviembre de 2018
}

\section{Resumen}

Para mejorar la igualdad de género, reducir daños de reputación en las organizaciones y mejorar su resiliencia, es necesario analizar su situación real y participar en un diálogo constructivo. Sin embargo, a menudo éstas carecen de la formación necesaria para implementar políticas exitosas con ese fin. Este artículo trata de cubrir dicho vacío proponiendo una herramienta para evaluar la inclusión de género actual en las CS. Se evalúan tres objetivos: 1) garantizar negocios con organizaciones que respeten la igualdad de género, 2) promover el emprendimiento femenino y 3) divulgar políticas de igualdad de género a lo largo de la CS.

\section{Palabras clave}

Cadena de Suministro; Resiliencia; Igualdad de Género.

\section{Introducción}

La gestión tradicional de la Cadena de Suministro (CS) se ha ampliado para considerar factores como la agilidad y resiliencia que permitan hacer frente a un entorno cambiante, y al mismo tiempo, las nuevas CS, han creado nuevas oportunidades de empleo para ambos géneros. A lo largo de la cadena de suministros se transmiten productos y servicios, pero también intangibles (como valores etc.) que sin ser su objetivo principal pueden tener impacto en el entorno. Desde este punto de vista se pretende analizar cómo la igualdad de género se transmite a través de la CS. Monitorizar el desempeño de género en toda la CS puede constituir una parte importante de la gestión de riesgo de las organizaciones, por ejemplo, al ayudar a evitar daños a su reputación mediante la asociación a condiciones de trabajo discriminatorias y precarias (Cuenca et al. 2017). Incorporar la perspectiva de género implica incluir no solo dimensiones vinculadas directamente a los eslabones de la CS, sino también el estudio

\footnotetext{
Llanos Cuenca *

1lcuenca@cigip.upv.es

https://orcid.org/0000-0003-3589-4182

Ana Esteso *

aneslva@doctor.upv.es

https://orcid.org/0000-0003-0379-8786

Elena Navarro-Astor $* *$

enavarro@omp.upv.es

https://orcid.org/0000-0002-1588-9186

* Centro de Investigación en Gestión e Ingeniería de la Producción (CIGIP). Universitat Politècnica de València. Camino de Vera S/N, 46022, Valencia

** Escuela Técnica Superior de Ingeniería de Edificación, Dpto. Organización de empresas. Universitat Politècnica de València. Camino de Vera S/n. 46022, Valencia
}

de las relaciones que se dan fuera de éstos (relaciones de género).

El análisis de género permite conocer las posiciones, condiciones y necesidades específicas de mujeres y hombres, su acceso al control de los recursos y al proceso de toma de decisiones. Se trata de una herramienta que permite comprender las diferentes necesidades de mujeres y hombres, y dar respuestas más eficientes, focalizadas y participativas (Escuela Virtual de Igualdad, 2016). Incluir el género en el análisis de una CS implica profundizar en los roles desempeñados por hombres y mujeres a lo largo de la cadena y en sus condiciones de trabajo, no solo con un fin descriptivo sino para comprender los procesos socioculturales, económicos e institucionales que llevan a su exclusión o inclusión en los diferentes eslabones (Alesina 2007). Si se establece una forma de analizar y medir la incorporación de la perspectiva de género en la cadena de suministro, se pueden establecer parámetros que faciliten su transmisión.

Por otra parte, las organizaciones actualmente se mueven en entornos de incertidumbre, están expuestas a situaciones imprevistas, amenazas y situaciones que provocan alteraciones en el desarrollo de su actividad y por tanto en su rendimiento empresarial. Es por todo ello, y debido a que los mercados son cambiantes y cada vez más exigentes, que las organizaciones precisan dar respuestas rápidas, para hacer frente a dichas situaciones.

Se define resiliencia como la habilidad de una empresa de cambiar, adaptarse y/o recuperarse rápidamente alineando su estrategia, procesos, tecnología y personas para la consecución de sus objetivos, la maximización de su desempeño y el aseguramiento de su capacidad de respuesta y adaptación a los continuos cambios (Sanchis and Poler 2011). La resiliencia es una característica obligatoria de la CS con el fin de proporcionar la capacidad de adaptarse a los cambios y de- 
sarrollarse en el largo plazo. Las organizaciones luchan por sobrevivir de las recesiones y las interrupciones, pero aquél más rápido en adaptarse no sólo sobrevive, sino que puede también dominar a sus competidores (Soni and Jain 2011).

En el contexto de CS, diversos autores han identificado factores que facilitan la resiliencia, como son: redundancia, flexibilidad, visibilidad y colaboración (Ali, Nagalingam, and Gurd 2017). No incorporar la perspectiva de género en el diseño de la CS, puede limitar los factores anteriores y por tanto reducir la resiliencia. Desafortunadamente las organizaciones muchas veces carecen de conciencia, herramientas y capacitación para implementar prácticas exitosas que incorporen la igualdad de género. En este sentido se pueden elaborar recomendaciones para analizar el impacto de la promoción de la igualdad de género en la CS y su impacto.

Tomando como referencia el informe de (Miles 2011; GRI \& IFC 2009); en (Cuenca et al. 2017) se propuso la definición preliminar de un modelo de madurez que permite evaluar la incorporación de la perspectiva de género en las organizaciones, sin limitar su agilidad y resiliencia. Para ello, se identificaron diferentes áreas clave donde considerar los aspectos de perspectiva de género, entre las que se encuentran la CS. Para esta área deberían cumplirse los siguientes objetivos: 1) Garantizar negocios ágiles y resilientes con organizaciones que respeten la igualdad de género. 2) Promover de forma ágil la actitud emprendedora femenina. 3) Divulgar informes sobre políticas de igualdad de género a todos los niveles de la cadena, y analizar cómo estos contribuyen a la agilidad y resiliencia de la misma. A partir de esta propuesta el presente trabajo trata de determinar cuáles serán los elementos a tener en cuenta en la CS que permitan alcanzar dichos objetivos. No se trata de reformular los conceptos básicos de la CS, sino de utilizarlos, para analizar, desde su perspectiva, un fenómeno que indudablemente preocupa en nuestra sociedad como es la igualdad de género y ver su posible impacto en la resiliencia de la CS.

\section{Cadenas de Suministro Resilientes}

Incorporar la perspectiva de género es un enfoque que permite evaluar cómo las políticas, programas, proyectos y actividades influyen en los hombres y las mujeres. Tiene en cuenta los roles basados en el género, las relaciones, los recursos, las necesidades sociales/económicas y otras limitaciones impuestas por la sociedad, la cultura o la etnia.

Como se ha indicado anteriormente, las cadenas de suministro deben cumplir una serie de objetivos para garantizar la igualdad de género. Esto implica que todos los comportamientos, aspiraciones y necesidades inherentes a los hombres y mujeres se consideren, valoren y repartan equitativamente, y que no dependa del hecho de que hayan nacido hombre o mujer.

Las organizaciones mantienen relaciones con diferentes empresas desarrollando alianzas y estrategias de colaboración. Incorporar la igualdad de género no supone únicamente establecer actuaciones en su ámbito organizativo interno sino también trasladar este compromiso a las empresas con las que interaccionan bien como competidoras o proveedoras, o incluso entidades públicas. El reconocimiento de empresas facilitará el desarrollo de estrategias comunes.

\subsection{Objetivo 1: Negocios ágiles y resilientes con organizaciones que respeten la igualdad de género.}

La integración de género ayuda a las organizaciones, a que sus proveedores comprendan mejor cómo administrar, medir y mejorar su desempeño en sostenibilidad de género, mejorando relaciones con ellos y clientes a su vez. La igualdad de género es importante para el desarrollo, porque cuando las mujeres tienen acceso a la educación, ingresos propios y participan en la toma de decisiones, las sociedades tienden a elegir mejor y a tener mejores resultados de políticas. Se dice que la igualdad de género se ha alcanzado cuando los hombres y las mujeres pueden acceder y disfrutar de las mismas recompensas, recursos y oportunidades. El objetivo es lograr resultados iguales en términos generales entre mujeres y hombres, y no exactamente el mismo resultado para todos los individuos (Australian Goverment 2016).

Resulta de interés conocer si el compromiso interno con la igualdad se traslada a la contratación y a las relaciones con otras organizaciones colaboradoras. Traspasar la frontera de la propia organización, incorporando la misma responsabilidad con el cumplimiento del principio de igualdad a las organizaciones con las que se trabaja tiene un efecto multiplicador del compromiso interno (Escuela Virtual de Igualdad 2016).

Las cuestiones que nos ayudan a medir el alcance de este objetivo (Tabla 1) han sido extraídas de Devaux et al. (2016), UniBrain (2015), Royal Tropical Institute, Faida Market Link, and International Institute of Rural Reconstruction (2006), and Royal Tropical Institute, Agri-ProFocus, and International Institute of Rural Reconstruction (2012): 
Tabla 1 Preguntas para evaluar la igualdad de género

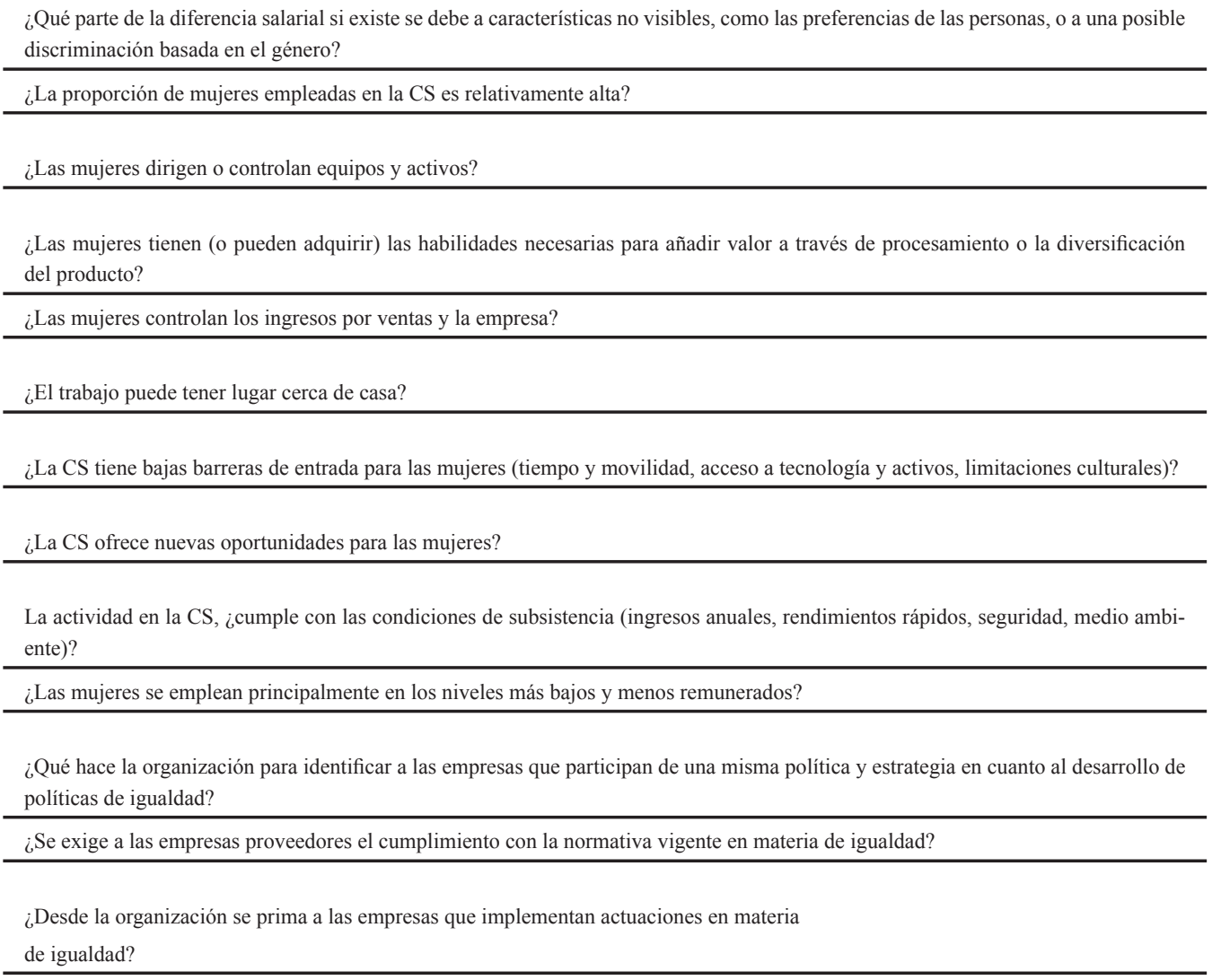

¿La proporción de mujeres empleadas en la CS es relativamente alta?

¿Las mujeres dirigen o controlan equipos y activos?

¿Las mujeres tienen (o pueden adquirir) las habilidades necesarias para añadir valor a través de procesamiento o la diversificació del producto?

¿Las mujeres controlan los ingresos por ventas y la empresa?

¿El trabajo puede tener lugar cerca de casa?

\subsection{Objetivo 2: Promover de forma ágil la acti- tud emprendedora femenina.}

Para alcanzar este objetivo, se debe invertir en políticas y programas que favorezcan el avance de las mujeres a todos los niveles y en todos los sectores económicos y que promuevan su acceso a todas las profesiones no tradicionales. Así mismo, las relaciones empresariales deben extenderse a las empresas dirigidas por mujeres, incluidas las pequeñas empresas (Moreno 2013). Las incubadoras de negocio pueden ayudar a esta tarea, como organizaciones diseñadas para acelerar el crecimiento y asegurar el éxito de proyectos emprendedores.

Las cuestiones que nos ayudan a medir el alcance de este objetivo (Tabla 2) han sido extraídas de Devaux et al. (2016), UniBrain (2015), Royal Tropical Institute, Faida Market Link, and International Institute of Rural Reconstruction (2006), and Royal Tropical Institute, Agri-ProFocus, and International Institute of Rural Reconstruction (2012): 
Tabla 2 Preguntas para evaluar la actitud emprendedora femenina
¿Las mujeres son miembros de la Junta Directiva de la incubadora de empresas si existe?

¿Las mujeres están presentes a nivel de toma de decisiones y gobierno del consorcio de incubadoras?

¿Están activamente involucradas en la toma de decisiones a nivel de incubadora?

¿Las mujeres que han sido preparadas en el programa de innovación, están en el mismo nivel que los hombres?

¿Las mujeres participan como propietarias de empresas o emprendedoras?

¿Las mujeres que se gradúan en el programa están en el mismo nivel que los hombres?

¿Existen oportunidades para la participación activa de mujeres?

¿Las mujeres se están beneficiando de las oportunidades de trabajo creadas por las empresas incubadas?

¿Los procesos de innovación se llevan a cabo colaborativamente?

Si la innovación es evidente, ¿quién gana con los resultados?

¿Existen mecanismos para compartir ganancias?

¿Se alienta a los pequeños productores a participar en la innovación inclusiva?

¿Hay muchas empresarias en la CS?

\subsection{Objetivo 3: Divulgar informes sobre políti- cas de igualdad de género a todos los nive- les de la cadena.}

El objetivo 3 supone transmitir el compromiso hacia la igualdad de género de la propia entidad, intercambiar con otras empresas las buenas prácticas realizadas en materia de igualdad de género y propiciar su incorporación en ellas.

Se alcanza teniendo en cuenta los impactos que todas las decisiones tienen sobre mujeres y hombres, ofreciendo condiciones de trabajo seguras y protección frente a la exposición a los materiales peligrosos, así como informando de todos los riesgos potenciales. Por otra parte, se debe asegurar la misma remuneración y los mismos beneficios por trabajo de igual valor y procurar pagar un salario mínimo vital a todos los hombres y mujeres; así como difundir las políticas empresariales y los planes de implementación a favor de la igualdad de género.

Las cuestiones que nos ayudan a medir el alcance de este objetivo (Tabla 3) han sido extraídas de Devaux et al. (2016), UniBrain (2015), Royal Tropical Institute, Faida Market Link, and International Institute of Rural Reconstruction (2006), and Royal Tropical Institute, Agri-ProFocus, and International Institute of Rural Reconstruction (2012): 
Llanos Cuenca et al. / Dirección y Organización 67 (2019) 52-58

Tabla 3 Preguntas para evaluar la difusión de las políticas de igualdad

¿Los actores de la CS comparten los mismos objetivos?

¿Los actores intercambian información regularmente?

¿Existen estructuras facilitadoras de la colaboración o resolución compartida de problemas?

¿Hay uno o más apoyos de la dirección que liderarán el proceso de innovación?

¿Todos los actores comprenden y reconocen la interdependencia de la relación comercial?

¿Los volúmenes y precios de compra-venta se comunican claramente?

¿Los estándares de calidad son claros y consistentes en toda la cadena?

¿Se comprenden y comparten los riesgos proporcionalmente a lo largo de la cadena?

¿Las relaciones comerciales se basan en contratos formales o en acuerdos informales claros?

¿Se han establecido indicadores que midan el éxito de la relación comercial?

¿Se miden con frecuencia los resultados de la relación comercial?

¿Existen circuitos de retroalimentación para garantizar una gestión efectiva de la cadena y la toma de decisiones?

\section{Aplicación al sector agrícola}

El sector agrícola está caracterizado por una gran cantidad de incertidumbres que impactan sobre su comportamiento y que generan la necesidad de diseñar y gestionar CS resilientes. Además, la sociedad está especialmente concienciada con respecto a la sostenibilidad del sector agroalimentario, donde se incluye el componente social de la perspectiva de género (Esteso, Alemany, and Ortiz 2018). Por ello, se decide aplicar la herramienta propuesta para evaluar la situación de la perspectiva de género en CSs a una CS del sector agrícola.
A partir de la recopilación de las cuestiones realizadas, para medir el cumplimiento de los objetivos anteriormente descritos, se procede a su validación sobre una CS del sector agrícola.

Tomando como referencia los niveles de alcance definidos en Cuenca et al. (2017) la organización analiza su nivel de madurez.
Figura 1 Niveles del modelo de madurez integrado para la agilidad, resiliencia y perspectiva de género (Cuenca et al., 2017)

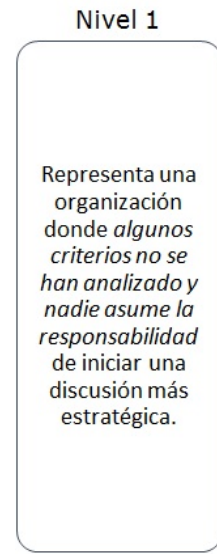

Nivel 2

\begin{tabular}{|l|}
\hline \\
La organización \\
comienza a \\
implementar \\
acciones para \\
alcanzar el \\
objetivo \\
marcado, sobre \\
una base bien \\
definida, pero \\
dejando a la \\
organización muy \\
dependiente de la \\
competencia de \\
los gestores del \\
sistema para cada \\
criterio.
\end{tabular}

Nivel 3

La organización
ha establecido un
enfoque
centralizadoy
coordinado para
alcanzar el
objetivo deseado.
Nombramiento
de un
administrador
central que
distribuirá
procedimientos
estándar. Tiene
roles y
responsabilidades
claramente
definidos.

Nivel 4

Incluye un enfoque coordinado, apoyado por una

mayor

recopilación de

datos. Requiere también mediciones

regularesy pruebascon escenarios de uso que puedan hacerlo estadísticamente

predecible y permitir mejoras estratégicas.
Nivel 5

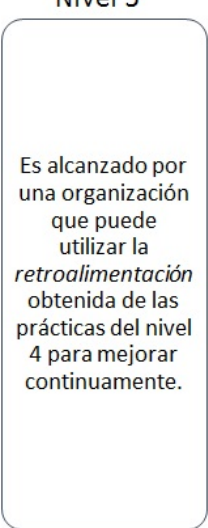


Los resultados obtenidos a través del modelo de madurez se pueden emplear para determinar cuáles son las mejores prácticas que las diferentes organizaciones deberían implementar con el objetivo de mejorar en cuanto a la perspectiva de género.
La Figura 2 muestra el resultado del análisis llevado a cabo sobre una cadena de suministro del sector agrícola utilizada para validar el modelo propuesto.
Figura 2 Aplicación del modelo de madurez

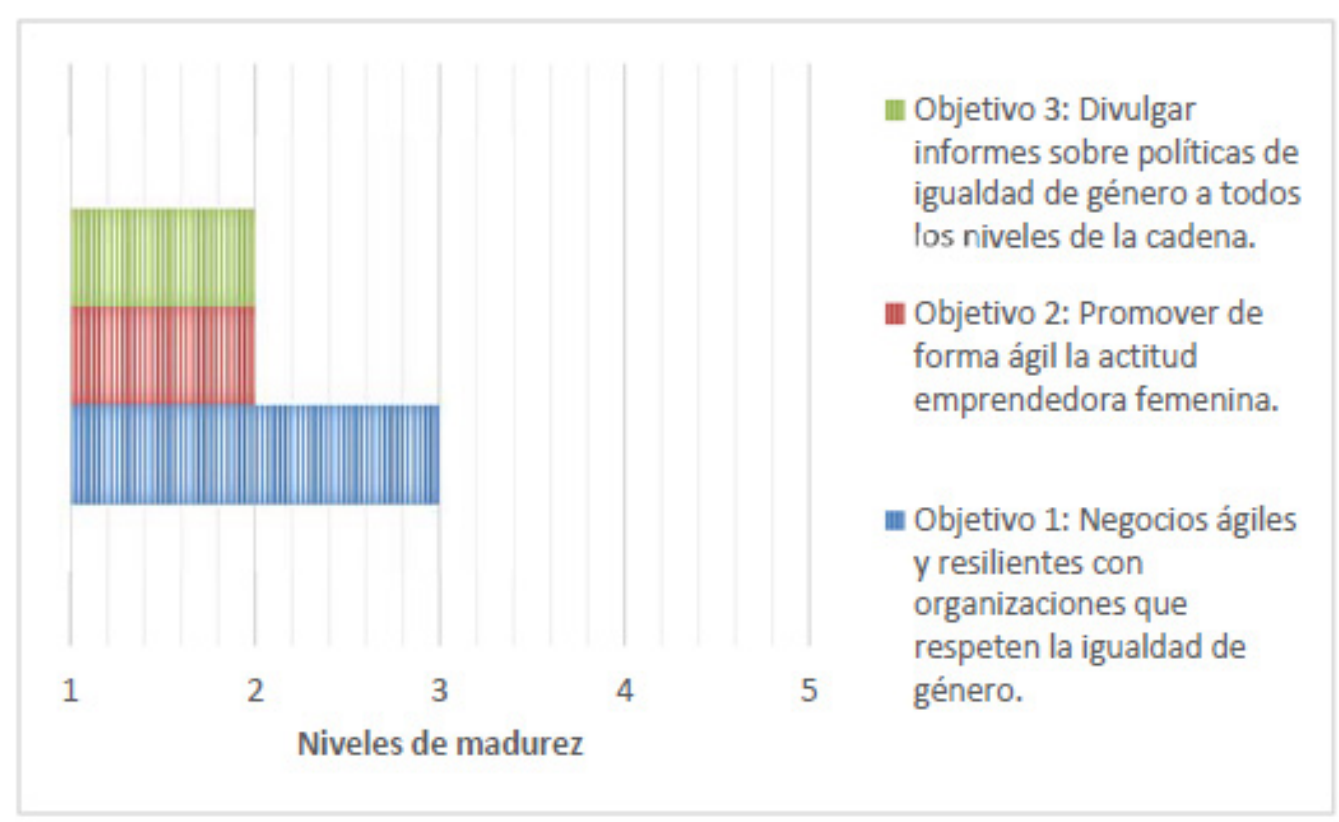

Los resultados indican un estado inicial de incorporación de los objetivos a alcanzar para la incorporación de la perspectiva de género en CS ágiles y resilientes. Se puede ver cómo la CSA ha comenzado a implementar acciones para alcanzar los objetivos 2 y 3 , dependiendo en gran medida de los gestores que se han identificado para ello. Lo que implica que los actores no intercambian información regularmente y no existen estructuras facilitadoras de la colaboración o resolución compartida de problemas. Los procesos de innovación no se llevan a cabo de forma colaborativa, y no se hacen mediciones regulares de los estándares de calidad.

Por otra parte, la organización ha establecido un enfoque centralizado y coordinado para alcanzar el objetivo 1, identificando los roles y responsabilidades que permitirán implementar actuaciones en materia de igualdad, y a través de un enfoque coordinado controlar la diferencias salariales y las barreras de entrada que puedan surgir.

\section{Conclusiones}

Estudios previos sobre la gestión de las cadenas de suministro han abordado el concepto de la sostenibilidad desde el punto de vista económico y medioambiental, mientras que el aspecto social apenas ha sido estudiado. Sin embargo, existe una demanda creciente para que las cadenas de suministro mejoren sus valores sociales, y fuertes presiones para evitar daños comerciales y de reputación relacionados con la discriminación, la explotación y condiciones de trabajo indecentes. Un factor importante de la sostenibilidad social es precisamente la igualdad de género. Así, monitorizar el desempeño de género a lo largo de la CS de las empresas se ha convertido en un factor importante de sostenibilidad social.

En este trabajo se profundiza en la CS como una de las áreas clave de un modelo de madurez que permite evaluar la perspectiva de género en las organizaciones, sin limitar su agilidad y resiliencia. Se plantean tres objetivos concretos que debería cumplir una CS resiliente y se proponen una serie de cuestiones útiles para hacer el diagnóstico de la situación de la empresa para cada objetivo. Además, se presenta un ejemplo de validación de la herramienta en una CS del sector agrícola. La herramienta resulta fácil de utilizar y los resultados son sencillos de interpretar para obtener conclusiones.

\section{Acknowledgements}

Esta investigación se ha llevado a cabo en el marco del proyecto "Desarrollo de un modelo de madurez integrado para la agilidad, resiliencia y perspectiva de género en cadenas de suministro (MoMARGE). Aplicación al sector agrícola." Ref. GV/2017/025 financiado por la Generalitat Valenciana. El tercer autor agradece la financiación parcial por el programa de Formación de Profesorado Universitario del Ministerio de Educación, Cultura y Deporte Español (FPU15/03595). 


\section{Referencias}

Alesina, Lorena. 2007. "Guía Metodológica Para El Estudio de Cadenas Productivas Con Perspectiva de Género." Resumen ejecutivo.

Ali, Imran, Sev Nagalingam, and Bruce Gurd. 2017. "Building Resilience in SMEs of Perishable Product Supply Chains: Enablers, Barriers and Risks." Production Planning and Control 28 (15): 1236-50. doi: 10.1080/09537287.2017.1362487.

Australian Goverment. 2016. "The Business Case for Gender Equality."

Cuenca, L., E. Navarro-Astor, M.M.E Alemany, and A. Boza. 2017. "Áreas Clave Para La Alineación de La Agilidad y Resiliencia Con La Perspectiva de Género En Las Organizaciones." Dirección y Organización 63: 57-64.

Devaux, André, Maximo Torero, Jason Donovan, and Douglas Horton. 2016. "Innovation for Inclusive Value Chain Development: Successes and Challenges." International Food Policy Research Institute (IFPRI). doi:10.2499/9780896292130

Escuela Virtual de Igualdad, 2016. Diseño y Aplicación de Planes y Medidas de Igualdad en las Empresas. Nivel Avanzado. $6^{\mathrm{a}}$ Edición. Instituto de la Mujer y para la Igualdad de Oportunidades. Ministerio de Sanidad, Servicios Sociales e Igualdad.

Esteso, A., M.M.E. Alemany, and A. Ortiz. 2018. “Conceptual Framework for Designing Agri-Food Supply Chains under Uncertainty by Mathematical Programming Models." International Journal of Production Research. doi: 10.1080/00207543.2018.1447706.
GRI \& IFC. 2009. "Embedding Gender in Sustainability Reporting A Practitioner's Guide."

Miles, Katherine. 2011. "Embedding Gender in Sustainability Reports." Sustainability Accounting, Management and Policy Journal, 2 (1): 139-146. doi: $10.1108 / 20408021111162164$.

Moreno, Carmen. 2013. "Prólogo RSE, PYMEs, e Igualdad de Género.” Organización de los Estados Americanos.

Royal Tropical Institute, (KIT), Agri-ProFocus, and (IIRR) International Institute of Rural Reconstruction. 2012. "Challenging chains to change."

Royal Tropical Institute, (KIT), Faida Market Link, and (IIRR) International Institute of Rural Reconstruction. 2006. "Chain Empowerment: Supporting African Farmers to Develop Markets."

Sanchis, Raquel, and Raúl Poler. 2011. "Medición de La Resiliencia Empresarial Ante Eventos Disruptivos. Una Revisión Del Estado Del Arte." In V international conference on industrial engineering and industrial management, 104-113.

Soni, Umang, and Vipul Jain. 2011. "Minimizing the Vulnerabilities of Supply Chain: A New Framework for Enhancing the Resilience." In 2011 IEEE International Conference on Industrial Engineering and Engineering Management, 933-39. IEEE. doi:10.1109/ IEEM.2011.6118053.

UniBrain. 2015. “A Toolkit for Gender Mainstreaming in Agribusiness Incubation." 